association analysis also demonstrates differential promoter methylation in the core circadian genes in breast cancer cases relative to controls. However, whether epigenetic changes associated increased breast cancer risk could be caused by circadian disruption through exposure to light at night have not been explored. The current pilot study aimed to investigate epigenetic impact of long term shift work and cancer relevance of identified genes with altered methylation patterns.

Methods Archived blood DNA samples of 117 female subjects from a prospective cohort conducted in Denmark were included in the study. PCR-based method was used for methylation detection of gene promoter, while genome-wide methylation analysis was performed on the Illumina Infinium Methylation Chip.

Results Long term shift work resulted in the same promoter hypomethylation in CLOCK and hypermethylation of CRY2 in PBLs as was previously observed in breast cancer cases. Genome-wide methylation analysis discovered widespread methylation alterations in shift workers, including changes of many methylation and cancer relevant transcripts. Ingenuity pathway analysis of the genes with altered methylation patterns further revealed several cancer-related pathways. One of the top networks generated was designated as "DNA replication, recombination, and repair, gene expression, behavior" with ESR1 featuring most prominently in the network.

Conclusions These findings, although exploratory, demonstrate the first evidence of cancer relevant epigenetic effects of night shift work, which warrant further confirmation and investigation. This work was supported by NIEHS grant ES018915 and funds from the Danish Cancer Society.

\section{GENOME-WIDE METHYLATION CHANGES IN NIGHT WORKING WOMEN IN DENMARK: SAME ALTERED PROMOTER METHYLATION OF CLOCK AND CRY2 AS IN BREAST CANCER CASES}

Yong Zhu, ${ }^{1}$ Richard Stevens, ${ }^{2}$ Aaron Hoffman, ${ }^{1}$ Anne Tjonneland, ${ }^{3}$ Ulla Vogal, ${ }^{4}$ Tongzhang Zheng, ${ }^{1}$ Johnni Hansen ${ }^{3}$ 'Yale University, New Haven, USA; ${ }^{2}$ University of Connecticut, Farmington, USA; ${ }^{3}$ Danish Cancer Society, Copenhagen, Denmark; ${ }^{4}$ National Research Center for the Working Environment, Copenhagen, Denmark

10.1136/oemed-2011-100382.56

Objectives A substantial body of literature has recently linked circadian disruption to breast cancer risk. Epigenetic 